\title{
Papers
}

\section{The business realities of metadata: What really matters when it comes to operations and commercialization of assets}

\author{
Pam Williams \\ is a consultant, operating in the field of digitization and commercialization of photography and footage collections. She \\ has worked in the media sector for over 25 years, specializing in data, imaging and production. She has held senior \\ operational roles at The Independent newspaper and most recently Getty Images.
}

Keywords: digital asset management, metadata, commercialization, relevant metadata, accurate metadata, consistent metadata

\begin{abstract}
Metadata are essential for a digital asset to have a viable shelf-life and future commercial value. They will also be key to the successful delivery of expected business efficiencies from the installation of a digital asset management system. This paper discusses the main operational metadata issues that need to be taken into account when either setting up a digital asset management system or analyzing an existing archive for potential commercialization.
\end{abstract}

\section{INTRODUCTION}

Without metadata an asset is of little commercial value beyond its immediate moment in time. Obviously there are some exceptions, but even a previously unknown photograph of, say, Marilyn Monroe would be less valuable without the context in which it was produced.

To be of any medium- or long-term value, an asset needs metadata and those metadata need to have four crucial attributes:

Pam Williams

Digital Content

Consultant

37 The Pines

Woodford Green

Essex IG8 ORW, UK

Tel: +44 (0) 208505

6737

Email: pam.williams99@

btinternet.com
- existence

- relevance

- accuracy

- consistency

\section{EXISTENCE}

To be of any use, and before any other attributes come into play, metadata need to exist! It sounds pretty obvious but every day valuable "commercial" assets are being created and/or archived without even a minimal amount of information.

Without metadata any asset will have a fairly short shelf life. The exact amount of time will depend on the unique nature of the document, photograph, footage etc. but the deterioration will begin within hours unless the photographer, author, designer or film maker either has total long-term recall (ie is superhuman), produces clear, structured records themselves, or passes on the necessary information for someone else to record. Just to be clear, although we tend to think of metadata as information stored in a database or digital asset management 
(DAM) system, they can of course be stored on paper, in log books etc. In the digital age this is unlikely to be the most useful or efficient method of long-term information recording, but many legacy archives rely on well-gathered and maintained paper-based metadata. There are several methods in which this information can be entered into a system as part of a DAM or commercialization project - the key point being that the information at least exists.

Imagine an entertainment photographic agency that has had access to major celebrities at high-end events over the last ten years. Many of the images sold well at the time and have faithfully been scanned and stored in a database - because we have all known for years that the future is digital. Unfortunately no information much beyond the photographer's name is entered because it would take too long and there is always the next job to do.

At some point in the future, one of the major photo distributors may come along to take a look at the collection with a view of purchasing the company or representing the agency. Or perhaps the agency owners are exploring other opportunities to commercialize their archive; after all, they have spent money scanning and retouching pictures, as well as purchasing and maintaining a system to house the digital images.

Unfortunately, without accurate information about each image, appropriate copyright information and perhaps licensing history, this collection will be of little commercial value.

It is unlikely that the photographer would remember the details even if they could be persuaded to take the time to add them so long after the event. What was the occasion? What date? (what year even?). What designer dress is Nicole Kidman wearing (actually, is that Nicole Kidman?). Was that before or after she married Tom Cruise? Careful research may answer some of these questions but imagine the time and cost involved in working back through an archive of even a few hundred pictures.

Instead of the opportunity for a new revenue stream at limited cost, as anticipated by the agency, considerable time and money would need to be invested in research and metadata addition to make the archive commercially viable.

From a business point of view, even this first step to metadata nirvana may provide some interesting challenges. Creators are notoriously reluctant to record and/or provide the information their "operational" colleagues require. It may be necessary, or even advisable, to ensure this is a contractual requirement if there is any kind of expectation that either commercial or cost-saving opportunities can be realized from digital archives. Obviously this should be predicated with good communication, information and explanation, and backed up at all stages with training and support.

Even if you can get the creators to play ball, you may still have senior managers and budget holders to convince. Despite their importance, metadata are often not understood by non-specialists. More often than not they are regarded as a drain on resources to be cut back and kept under control not recognized as a key element to successful commercialization and asset management and a crucial value-add to the enterprise. Try to find imaginative and non-taxing ways to inform these key stakeholders and demonstrate the 
benefits of good metadata and their role in the overall asset management strategy.

The worst possible situation to find yourself in is a requirement to add significant amounts of data to an archive retrospectively. The pain of doing this is significantly worse if the information could, or should, have been entered as part of ongoing data operations. But even that is better than finding yourself responsible for an archive of little or no use due to the lack of information relating to the assets.

\section{RELEVANCE}

The scale and structure of metadata that needs to be applied will very much depend on the exact circumstances of the enterprise. It is essential that careful consideration and research is carried out at the planning stage to determine the real metadata needs. Too little data may make the assets worthless - too much data will cost the organization time and money with no likely return. Some key areas that will need to be considered are detailed below.

\section{The system/search engine to be used}

It is essential that the technologists and the business experts work closely here. With a new installation of DAM there is an opportunity - in fact it is a crucial part of the exercise - to analyze all the factors that will impact the metadata. What data are needed, how they will be entered and how the end user will interact with the system and assets are very key considerations. All DAM solutions are not equal and do not all work in the same way. As with any system installation or change, thorough analysis and planning upfront will be crucial to success.
Whether a new installation or an existing system, there will be circumstances and limitations that must be identified and worked with. It is essential that these be understood before investment in time and resources is made applying data that cannot be utilized by the chosen system, or customer/ management expectations are raised that cannot be met by existing infrastructure regardless of the richness of available metadata.

A system may operate on keyword searching; but does it support a tree structure or will every asset be keyworded according to the mood or inclination of an individual keyworder? (Does the image depict a girl on a beach, a woman by the sea or a female on the sand?) Will there be a thesaurus and/or synonym dictionary and how easily can that be developed and maintained? Is there a limit on the number of fields that can be used or customized? Is it possible to determine primary and secondary data so that the most relevant material will be returned first?

Free text searching may be more appropriate to some organizations - it will drive different metadata requirements to keyword searching and require clear operating standards, a style guide and consistent training with regular reviews to maintain quality search results. Consider sending the metadata editors on a copywriting course to sharpen up their prose and develop a consistent style.

If you have only free text available and need keyword data it may be possible to make use of text segmentation software. This will pull the keywords out from the text to give a reasonable starting point for a keyword structure. A good metadata specialist 
should then be able to review the output of text segmentation and, using techniques such as data mapping, identify items which can be adapted in bulk, those which may need additional work and areas in any existing metadata schema which need adapting to accommodate new material.

\section{Numbering schemas}

It will almost certainly be the case that a new DAM system will apply a unique identifying number to each asset. Don't fall into the trap of just renumbering all existing material. Legacy systems, processes, descriptions applied to assets; all these will probably have original numbers to which people will continue to refer. Make sure that for any legacy material migrated into a new system, legacy numbers or other descriptions are maintained as part of the metadata.

In addition, be very careful about applying numbering schemas with builtin intelligence; that is the role of metadata not unique identifying numbers. I once came across a production unit that had digitized the entire image collection of a travel company using the page number and position on the page from the most recent brochure as a unique number. The new "digital workflow" process unraveled very quickly as designers and editors could not identify the images they needed for the new publications there was no such thing as a standard brochure as the designs changed regularly!

\section{Amount of detail}

If it is commercially appropriate to give the fullest possible level of description, it may be wise to define primary and secondary level data. There are no hard and fast rules of what is right and what is wrong with regard to the level of metadata applied - this is an area where decisions must be appropriate for the business or department using the assets. I would however suggest that medium- to long-term business and operational objectives are understood before any particular data are ruled in or out. For example, a marketing department expecting their company to face demerger or re-branding in the next few months should probably take account of that when determining metadata issues as it may have significant impact on future use of the material in their archive.

\section{Customer expectation}

Whether internal or external customers (or both), how are users going to interact with the system or the assets and what will their expectations be? Metadata cannot simply be dreamt up in glorious isolation. No matter how strongly metadata editors feel about the use of particular language, it is customer/user behavior that will determine whether the metadata "works." As well as the language and data structure being user-friendly, do the metadata provide users with the information they are looking for? If a key customer concern is date of authorship, but the system only records the date when the item is loaded into the system, the result will be unhappy users and dissatisfaction with the system.

\section{Future product development}

If the company has strategic ambitions to move into another area of business, will the assets currently being archived need to be presented in a different way, with different or additional information? 
Evaluate whether or not additional metadata to meet this need should be collected/entered initially.

\section{Industry standards}

Do any industry standards apply in the case of your particular enterprise or organization? If so, should you work with them or strive out for something new that will give you a competitive advantage?

\section{Future users}

It is usually wise to think through realistic future uses of the material. For example, if a DAM system is being installed for use by the marketing department, but the expectation is that 12 months down the line the production department will use the system, it may make sense to ensure data recorded from day one will meet the likely needs of both departments. Again, the operational checks and balances will vary for every installation and organization, but don't be caught out by not even considering them.

\section{Localization}

Most of us are operating in a global economy. It may be that an initial project is not relevant outside the home territory, but if there is any expectation or reasonable likelihood that this will be a future requirement, make sure there is a strategy for dealing with localization of metadata. Careful planning upfront can prevent expensive reworking as a site or system is rolled out to other territories.

\section{UPDATING}

One of the most common mistakes is to assume that once a metadata record has been created there will be no need to change it. But of course, language is a living, changing thing and any number of issues, from world events like September 11th and the Tsunami to television programs like Big Brother and Sex and the City can and do impact language and how and what users search for or need to retrieve. Rapidly changing legal guidance on rights, trademarks etc may also impact how assets can and should be described. The really good metadata managers ensure records are updated as appropriate. For this not to become an operational nightmare it is important that the systems being used allow metadata records to be added, deleted, edited or appended to in bulk — changes of this nature rarely, if ever, impact just one record.

\section{ACCURACY}

Once the business requirements for metadata have been determined and there is a blueprint for the relevant data which needs to be recorded, it is essential to put systems, people and processes in place to ensure the accuracy of the data. It is pointless to record information that is inaccurate and there could be serious commercial consequences arising from data errors.

Depending on the system or search engine used, the asset inaccurately tagged may simply never be found again. All other investment in that material will be wasted and it may need to be re-created. One of the return on investment cases invariably used to justify the installation of a DAM system is the reuse of existing material; this will rapidly become an embarrassment if assets cannot be found.

An asset that is going to be supplied on a commercial basis must be fit for purpose like any other product. Information attached to the asset, eg 
usage rights, copyright, descriptive data - in fact any information that can be seen and/or used by the customer must either be accurate or supplied with the caveat that it may not be.

A publisher printing an image captioned "Tree Frog (Phyllomedusa vaillanti)" in their latest wildlife reference book is not going to be very happy if the frog in question turns out to be, in fact, an Asian Leaf Frog (Megophrys montana). It may be difficult for you or me to get excited about something like that - but like beauty, accuracy is in the eye of the beholder. If you don't want to take responsibility for information to that level of detail, make sure your customers (whether internal or external) are aware that they have the responsibility to research themselves.

In the average operation, responsibility for the accuracy of metadata is effectively going to end up in the hands of a metadata editor, keyworder, indexer, or whatever job title that particular organization has plumped for. This inevitable loss of direct management control means that the selection, motivation and training of staff is extremely important. I am sure we have all heard horror stories of disaffected data editors entering rude words; however, the most likely cause of poor quality data is the lack of proper, written guidelines, initial and ongoing training and an appropriate level of quality control.

It is normally true that the creator of an asset is not the best person to then apply metadata - there may be some exceptions but that is a general rule. However, for the very best records, a close relationship between creators and metadata editors is essential. The transfer of information works both ways. It is important that creators pass on accurate, timely information in the most usable format for ongoing metadata records to be entered - but it is often also very valuable for the data editors to understand some of the thinking behind the creative process. Regular, organized, constructive dialogue between creators and metadata editors is invaluable.

Without this communication and respect poor quality data are likely to result and relationships will be strained and feature constant complaint and mistrust.

There will be significant operational efficiency and improved usability and quality if controlled fields and vocabulary, drop-downs etc can be used in the DAM system. Any completely free application of data will lead to variances and should be avoided. If it is not possible to have system-driven limitations (such as selection from drop-down menus) the level of training, ongoing review, quality control checks, updating of procedure manuals etc will need to be greater.

\section{CONSISTENCY}

Closely related to accuracy is consistency. Hopefully in the internet age, where the importance of searching, at least, is obvious, metadata experts are more respected than in the past. But there is still a tendency in organizations for "data production" to be passed around from department to department, one year reporting to Marketing, the next to Technology, the third bundled into a new operations functions following a reorganization - and so on. Clearly the senior managers from such diverse disciplines are likely to have very different priorities and points of view. This lack of consistent direction can be very damaging to the objective of maintaining standards. However 
relevant and accurate metadata are, if they have not been applied consistently the value of the entire archive is compromised.

Such scenarios may be difficult to avoid. Whether or not it is likely to be an issue in your organization, there are some obvious steps which may limit the damage if it does happen - and is not wasted activity even if it doesn't. I would always recommend ensuring that key metadata experts are visible to the appropriate stakeholders in the organization. It is important that they don't shroud the work of their team in technical or academic language, which will turn people off. It is also advisable that they regularly communicate with all departments to ensure the relevance of their work across the organization. In the event of an organizational change, there will hopefully be sufficient understanding and respect for the work of the metadata team and the value of a consistent approach that the risks of moving to a different function will be limited.

As mentioned above with relation to accuracy, the design of the system, selection of the right staff, regular training and team communication, and production of user-friendly procedure manuals are crucial to the delivery of consistent metadata. Without these tools and good management practices you are setting up your metadata editors to fail, no matter how dedicated they are.

\section{CONCLUSION}

It is important for any organization or enterprise embarking on DAM to be aware of the importance of metadata and the people who are responsible for them. Without appropriate metadata you do not have an asset of any longterm value.
Every organization or department will have different reasons for running a DAM system; those reasons (end use, lifecycle, scope of current and future projects, customer expectations, business model etc) must inform and determine the type and scale of metadata collected and its ongoing management. Wherever possible, find out what other organizations in your field are doing, work with industry standards wherever they exist, but do not follow these guidelines slavishly if they don't work for your needs. If an element of data is of relevance to your organization, don't fail to collect it because no one else does.

It is arguably worse to attach bad, inaccurate data to assets than to attach none at all. It can be difficult to imagine all the possible consequences, but normally assets are stored in a DAM system because they have some ongoing value related to their specific properties. Inaccurate data may damage or destroy those properties and render the item valueless. Even worse, commercial damage may be caused because the wrong asset is used due to inaccuracy. Metadata handling is not for the careless and slipshod.

Finally, a DAM system is like any other database - garbage in, garbage out. Once the "rules of engagement" for the nature and structure of the metadata are established, these must be applied consistently. This is not the kind of operation where you can get away with diving in "just to get started," fathom out the rules as you go along, and change things that don't work later down the line. Your assets will be of limited value without consistent management of metadata.

(C) Pam Williams 